\title{
LA ACTUALIDAD CONSTITUCIONAL EN ESTADOS UNIDOS EN 1997
}

\author{
MARCOS CRIADO Y ANTONIO DE CABO \\ Universidad Complutense
}

En el presente trabajo se aborda, desde dos puntos de vista, la problemática constitucional de los Estados Unidos de América en el año 1997. Estas dos perspectivas son: el debate doctrinal entre los constitucionalistas en relación con las modificaciones legislativas y políticas con relevancia constitucional, y la labor de los Tribunales en estas mismas áreas. Conviene, sin embargo, precisar esta afirmación inicial. De un lado, tratándose de la primera revisión de este tipo, se desborda parcialmente el ámbito temporal del año 1997, para referirse a ciertos acontecimientos anteriores y, sobre todo, para describir la situación inicial sobre la que se ha producido el debate a lo largo del citado año. De otro, se tratan también asuntos que, desde la perspectiva española, quizá tendrían su encaje en el Derecho Administrativo. En relación con esta circunstancia, conviene recordar el hecho de que, por la propia naturaleza del derecho anglosajón, la diferencia entre lo que en el Continente se consideran áreas estancas se encuentra sumamente debilitada en los Estados Unidos; junto a ello, el Estado, como sujeto natural de la reflexión constitucional, está desarrollando su actividad de forma fundamental a través de las llamadas agencias, cuyo estatuto jurídico aunque presuntamente administrativo, ha conllevado la realización de numerosas tareas (normativas y de ejecución), semejantes a las que en España, por ejemplo, realizan ciertos "Örganos constitucionales" (como, en parte, se argumentará después). Por último, no se trata más que por referencia el debate suscitado por la publicación de la última obra de Dworkin, ello porque dicho debate está claramente abierto y su alcance real exige considerar su prolongación en el año 1998, reservándose su comentario, por tanto, para futuras revisiones (sin negar un inconveniente meramente material como es el retraso con que se reciben las revistas norteamericanas en nuestras instituciones universitarias).

UNED. Teoria y Realidad Constitucional, núm. 1, 1." semestre 1998, pp. 239-252 
Muy en consonancia con el tradicional pragmatismo norteamericano, el debate doctrinal a lo largo del año 1997 se ha centrado en los efectos derivados del programa de reducción del déficit público, con importantes recortes en los presupuestos de las agencias y una enorme reducción del número de empleados públicos. Las discusiones que ha suscitado esta política de austeridad presupuestaria se refieren tanto a la reforma de los medios de actuación de las agencias, como al ámbito de la judicial review. Como se verá, después, esta reducción presupuestaria no tiene un reflejo meramente organizativo, sino que afecta, sobre todo, a la eficacia de los Derechos de Prestación (derecho a la educación) y a la organización de la protección social. Es decir, a la naturaleza misma del Estado como Estado de Bienestar (the era of big government is over).

Un segundo foco de atención para la doctrina norteamericana en el año 1997, surge de la nueva regulación del habeas corpus introducida por la Antiterrorism Death Penalty Act (AEDPA) en el ámbito de la judicial review de las sentencias capitales condenatorias de los tribunales de los estados por parte de los tribunales federales. El debate se ha centrado, fundamentalmente, en las implicaciones constitucionales de la reforma, así como en la política de constitutional avoidance llevada a cabo por los tribunales federales en los últimos dos años y respaldada por la Corte Suprema en su sentencia Felker v. Turpin.

Como ya se ha dicho, por último, en 1997 se produjeron las primeras manifestaciones de un incipiente debate iusnaturalista en torno a la constitución norteamericana, generado por el libro de Ronald Dworkin: "Freedom"s Law: The Moral Reading of the American Constitution", cuyo comentario en profundidad escapa al ámbito temporal cubierto por este artículo'.

\section{"THE ERA OF THE BIG GOVERNMENT IS OVER" ${ }^{2}$}

Para centrar lo que ha sido el debate provocado por la ideología y práctica que sustentan las citadas palabras del Presidente B. Clinton, quizá convenga recordar cuáles eran las características esenciales del funcionamiento de la Administración norteamericana hasta la llegada de esta política de austeridad ${ }^{3}$. Como se indicaba al comienzo, la actuación estatal se ha ido desviando hacia las agencias en un proceso ininterrumpido de delegación de poderes en el que, desde 1935 (Panama Refining Co. v. Ryan, 293 U.S. 388 (1935)), el Tribunal Supremo no ha invalidado norma delegante alguna proveniente del legislador federal. Efectivamente, el Tribunal Supremo ha fijado un estándar de revisión extraordinariamen-

1. Dworkin, Ronald: Freedom's Law: The Moral Reading of the American Constitution, Cambridge, Mass., Harvard University Press, 1996. Puede consultarse una reseña en el Vol. 50, núm. 2, de la Stand. L. Rev., enero 1998.

2 Palabras de Bil. Cuinton en el Discurso sobre el Estado de la Unión en 1996.

3. Se sigue aquí, esencialmente, el resumen de BerNaki) SCHWArTZ: Administrative Law Cases during 1996, Adm. L. Reu., vol. 49.3, 1997. 
te bajo, el llamado "intelligible principle" (Mistretta v. United States, 488 U.S. 361, 372 (1989)), definido por uno de los magistrados del propio Tribunal (Judge Learned Hand) como la existencia de una mera "pista" sobre el alcance de la delegación. Esta capacidad de delegación no exigía un estándar más alto en ningún área e incluía la posibilidad de delegación en materia tributaria (Skinner v. Mid-America Pipeline Co., 490 U.S. 212 (1989)), o la tipificación penal incluyendo las agravantes que conducen a la imposición de la pena de muerte (Loving $v$. United States, 116 S. Ct. 1737, 1748 (1996)). En esta última sentencia se llegaba, de facto, a la supresión de estándar alguno de revisión para ciertos casos. Existía, pues, una consonancia total entre la política legislativa de delegación, el deseo presidencial de actuar a través de agencias y la interpretación que de las normas de delegación hacian los Tribunales (si bien es cierto que ciertos tribunales federales inferiores sostuvieron posiciones discrepantes nunca recogidas por el Tribunal Supremo). En la delegación se incluye no sólo la capacidad de realizar actos administrativos (adjudication), sino, si así se recoge en la ley, la capacidad de dictar normas (rulemaking), capacidad ésta controlada por los tribunales en la medida en que se ejerza ultra vires. Estas agencias tienen su propio sistema "quasi judicialn, siguiendo lo dispuesto en la Federal Administrative Procedure Act que pretendía crear una jurisdicción administrativa independiente, comparable a la de la justicia tradicional.

El reforzamiento de la capacidad de actuación de estas agencias (hasta casi su irresponsabilidad) producida por dos tendencias diferentes llegó a su punto más alto en 1996. De un lado, la capacidad de investigación de estas agencias que si hasta los años 40 exigía para la validez de un administrative subpoena que se tratase de encontrar pruebas de un incumplimiento específico de la ley, ha ido modificándose a través de diferentes decisiones judiciales (Endicott Johnson Corp. v. Perkins, 317 U.S. 501 (1943), United States v. Morton Salt Co., 338 U.S. 632 (1950)) hasta la situación de 1996 en la que, según el First Circuit, incluso tratándose de documentos de individuos y no de sociedades, bastaba con una indicación de que los materiales requeridos son a su [de la agencia] juicio razonablemente relevantes para su investigación" (Gimbel, 77 F.3d at 600). De otro, la llamada doctrina Chevron"t (Chervon U.S.A., Inc. v. Natural Resources Defense Council, 467 U.S. 837 (1984)), según la cual los tribunales debían practicar la deferencia judicial respecto de la interpretación que las agencias hicieran de las leyes

4. Sobre la doctrina Chevron Ver : Bernard Schwatz, Shooting the Piano Player? Justice Scalia and Administrative Law, Adm. L. Rev., vol. 47.1, winter 1995, págs.2 y ss. Apotbeosis of Mediocrity? The Rebnquist Court and Administrative. Law, Adm. L. Rev., vol. 46.2, 1994, Págs. 168 y ss. T. W. Merril.M, Textualism and the future of the Cheuron Doctrine, Wash. Un. L. Quartely, vol. 72, spring 1994. Robin CHARLow, Judicial Review, Equal Protection and the Problem with Plebiscites, Corn. L. Rev., vol. 79,1994 , Págs. 574 y ss.

Para el problema del control presidencial de las agencias ver : Steven G. Calabresi, Some Normative Arguments for the Unitary Executive, Ark. L. Rev., vol. 48, 1995, págs. 23y ss. LAw'RENCE Lessic \& Cass R. Sunstein, The President and the Administration, Colum. L. Rev., vol. 94, 1994, págs. 1 y ss. MICHAEl A. FITTS, The Paradox of Pouer in the Modern State: Why a Unitary, Centralized Presidency May Not Exbibit Effective or Legitimutte Leadersbip, U. Pa. L. Rev., num. 144, págs. 827 y ss, 1996. MARTIN FLAHERTY, The Most Dangerous Branch, Yale L. J., vol. 105, págs. 1725 y ss, 1996. 
para controlar su capacidad de dictar normas. Esta deferencia se ha aplicado, posteriormente, no sólo a la capacidad de dictar normas sino a cualquier actuación de las agencias, culminando en Smiley v. Citibank (116 S. Ct. 1730 (1996)), conforme al cual se denegaba la revisión judicial de una decisión de una agencia pese a que ésta había interpretado un término de la ley (interest) de forma contraria a como lo hacen todos los diccionarios, leyes y precedentes. En la práctica, suponía la práctica destrucción del enfoque tradicional de la revisión judicial de la actividad estatal.

Es, pues, frente a esta situación de reforzamiento permanente de la actuación de las agencias como hay que situar tanto la política legislativa del 104 Congreso, como las directrices del Contrato con América republicano que guió sus debates y que serían analizadas en el Simposium sobre el Futuro del Proceso Administrativo Norteamericanos.

Parece existir consenso doctrinal en que los recortes y congelaciones presupuestarias que la Administración Clinton ha llevado a cabo en los recursos de muchas agencias están en la base de las reformas producidas en los últimos dos años ${ }^{6}$. Ahora bien, el problema estriba en que no parece probable que esta reducción de los recursos lleve aparejada una correlativa reducción por parte del Congreso de las funciones y responsabilidades atribuidas a las agencias en sus respectivas leyes reguladoras. Es decir, que si, por una parte, la retórica política sigue asegurando al ciudadano el más alto nivel de protección, por otra, existe la decisión de cuadrar el déficit presupuestario en siete años y recortar los impuestos para las clases medias. De 1992 a 1996 se ha producido una reducción del número de empleados públicos en 250.000 personas, reducción que se agudizará en el período 1996-20027.

Así, en ausencia de una intervención legislativa que readapte las funciones de las agencias a su nueva y disminuida capacidad financiera, parece dejarse en manos de los jueces la resolución de los problemas derivados del progresivo

5. Recogido en Adm. L. Rev., vol. 49.1, Winter 1997, págs. 149 y ss. The Future of the American Administrative Process: Thomas O. Sargentich, The Question of the Future of the Administrative Process; JefrreY S. Lubbers, The Administrative Agenda for the Next Decade; William F. Funk, To Preserve Meaningful Judicial Review; CYNTHIA R. FARINA, The "Chief Executive" and the Quit Revolution ; ANDREW" F. Popper, Administrative Law in the 21st Century; T. AlexAnder AlinIKoff, Non-Judicial Checks on Agency Actions. El Contrato con América tenía dos objetivos principales: la devolución de competencias a los estados y el desmantelamiento del amplio sistema de protección social existente, sustituyéndolo por acciones concretas enmarcadas en un sistema de beneficencia pública. Todo ello se veria acompañado de un amplio programa de privatizaciones.

6 La futura reforma de la Occupational Safety and Health Administration (OSA) puede consultarse en un artículo de SIDNEY A. SAPHIRO y RANDY S. RABINOwITZ: Punisbment versus Coperation in Regulatory Enforcemente: A Case Study of OSHA, Adm. L. Rev., vol. 49.4, Fall 1997, basado en el report que los mismos autores realizaron como consultores de la OSHA. Ver también SIDNEY A. SAPHIRO, Substantive Reform, Judicial Review, and Agency Resources: OSHA as a Case to Study, Adm. L. Rev., vol. 49.3, Summer 1997, pág 645 y ss, aunque este artículo será analizado en el apartado actual.

La reforma de la Americans with disabilities Act merece también la atención del número de enero de The Yale Law Journal, vol. 107.4, Págs. 875 y ss, así como, unida a un análisis de la Discriminatory State Action, de la Harvard Civil Rights- Civil Liberties Law Review, vol. 33.1, Winter 1998.

7. Richard J. Pierce Jr, Judicial Review of Agency Actions in a Period of Diminishing Agency Resources, Adm. L. Rev., vol. 49.1, Winter 1997, págs. 66. 
incremento de la desproporción entre, de un lado, las competencias y responsabilidades administrativas de las agencias, y, de otro, los recursos de que disponen.

Ante esta responsabilidad, algunos jueces, respaldados por ciertas sentencias del Tribunal Supremo ${ }^{8}$, han respondido mediante una reformulación más relajada de las doctrinas ordinariamente aplicables a la judicial review de la acción administrativa. Otros, en cambio, consideran inapropiado considerar la reducción presupuestaria de la agencias en el proceso de aplicación de los principios legales administrativos?.

Veamos, pues, las posiciones doctrinales sostenidas en relación con este problema ${ }^{10}$. Richard J. Pierce Jr, frente a la posibilidad de que los jueces se arroguen competencias de decisión sobre la suerte de la regulación administrativa vía ajuste de las doctrinas sobre la discrecionalidad, prefiere la vía legislativa, o incluso la desregulación de las agencias ${ }^{11}$ y una mayor deferencia judicial hacia la actividad de las mismas.

Sidney A. Saphiro ${ }^{12}$, por su parte, no cree que las reformas legislativas, o ciertos procesos desreguladores, ayuden a reducir las carga económicas que las agencias suponen para el gobierno. Basándose en el caso de la OSHA, cuya reforma se está gestando, y como consultor de la Agencia en este proceso, formula algunas matizaciones a las soluciones propuestas por el profesor Pierce. En su opinión, los procesos desreguladores en la actividad legislativa de las agencias norteamericanas para asegurar espacios de absoluto libre mercado, han sido beneficiosos, por ejemplo, en áreas como los transportes por carretera de personas, pero han producido, en cambio, importantes problemas de competencia en el mercado de las líneas aéreas, como el propio Saphiro puso de manifiesto en 1993 en su libro "Regulatory Law and Policy". En esta misma línea, Susan Rose Ackerman ya advertía que: "Deregulation of one area of the economy may itself produce the need for regulation somewhere else. In moving toward a more competitive situation in one dimension, bottlenecks and other market imperfections in other dimensions may become newly relevant, ${ }^{13}$.

8. Por ejemplo, Pension Benefit Guard Corp. V. LTV Corp., 496 U.S. 633, 646 (1990) : "...if agency action may be disturbed whenever a reviewing court is able to point to an argually relevant statutory policy that was not explicy considered, then a very large number of agency decisions might be open to judicial invalidation."

9. El juez Richard A. Preston, en la sentencia del caso Salameda v. INS, 70 F.3d, determina : "understaffing is not a defense to a violation of administrative law". Citado por RiCHARD J. PIERCE Jr, op. cit., pág. 64 .

10 Este debate fue, en realidad, abierto algunos años antes de que el problema se hiciera acuciante con el libro de Stephen Breyer, "Breaking the vicious Circle : Toward effective Risk Regulation", citado por todos los autores que aquí se mencionan. Otros artículos publicados en los últimos anos sobre el tema y cuyas opiniones han influido en este debate son: CASS R. Sunstein, Congress, Constitutional Moments, and the Cost-Benefit State, Stan. L. Rev., vol. 48, págs. 247-287, 1996. Richard J. Pifrce Jr, Seven Ways to deossify Agency Rule Making, Adm. L. Rev., Vol. 48.1, Winter 1995.

11. "Desregulation or regulatory reform could offset, or more than offset, the dramatic reduction in agency funding that will take place over the next seven years." RicHaRD J. PIERCER Jr, op. cit., pág 67.

12. SIDNEY A. SAPHIRO, Substantive Reform, Judicial Revieu, and Agency Resources: OSHA as a Case to Study, Adm L. Rev., vol. 49.3, Summer 1997, págs. 645 y ss.

13. Susan Rose Ackerman, Rethinking the Progressive Agenda : The Reform of the American Regulatory State 153 (1992). 
Otra línea de actuación que contemplan estos artículos sería el cambio en los métodos de reglamentación y ejecución empleados por las agencias. Las posturas son dispares ${ }^{14}$. Puede decirse que existen dos opciones: bien realizar estrictas planificaciones anuales de prioridades que afronten de forma realista los recortes presupuestarios, y fijar, posteriormente, una regulación de los objetivos que deben conseguir los particulares conforme al método que ellos mismos elijan; bien regular exhaustivamente la materia, fijando no sólo los objetivos, sino también los procesos tasados para lograrlos. Gran parte de la actual doctrina parece abogar por la utilización de estándares de actuación como la forma más económica de regulación administrativa ${ }^{15}$. Es decir, por la fijación de formas de regulación más flexibles que permitan a la comunidad elegir los medios de actuación menos costosos para la consecución de los objetivos públicos. Ello se conseguiría creando estándares de actuación, o bien promoviendo niveles razonables de consecución de objetivos públicos frente a una regulación exhaustiva.

En lo que se refiere, concretamente, a la labor de los tribunales de revisión de la actuación administrativa en esta nueva fase de "Subfinanciación", el artículo del profesor Richard J. Pierce ha levantado cierto revuelo doctrinal entre los autores menos conservadores desde el momento en que propone una reducción de la capacidad de los jueces para revisar la actuación administrativa, reducción paralela a la que se da en los presupuestos y personal de las agencias, y que está previsto se incremente hasta el año 2002. Su argumento principal es que al aplicar la actual doctrina legal sobre el tema, las agencias deben desviar considerables fondos de otras actividades para cumplir con las determinaciones judiciales. Esta actitud fue brillantemente contestada por Thomas O. Sargentich ${ }^{16 .}$

También Cass R. Sunstein apunta la contradicción de esta doctrina: "Un principio fundamental del constitucionalismo americano consiste en que aquellos que están limitados por el Derecho no pueden decidir sobre el alcance de tal limitación" "17. La doctrina Chevron y su evolución posterior rompería este principio al permitir a las agencias interpretar el derecho que limita y controla su autoridad.

Pero el profesor Richard J. Pierce va más allá, pues, tras criticar el moderno sistema de reasonableness review, argumenta que una revisión centralizada en la cabeza del ejecutivo de las políticas de las agencias, reportaría más beneficios sin incurrir en los costes del actual sistema de revisión judicial. Y ello fundamentalmente por dos razones: en primer lugar, al igual que el juez Stephen Breyer, considera que los jueces no pueden entender los complejos procesos técnicos con los que las agencias toman sus decisiones, sirviéndose únicamente de las aportacio-

14. Un magnifico resumen de las mismas se encuentra en THOMAs O. McGariTr; The Expanded Debate over the Future of the Regulatory State, U. Chi. L. Rev., vol. 63, 1996, pangs. 1463-91.

15. Por ejemplo, a favor estaría R.J. PIERCE, op. cit., págs. 72 y 73 y, con reservas, SApHIRo, op. cit., pág. 650. En contra de una utilización excesiva ver, CAss R. Sustein, op. cit., pág 253, y William L. Dean, Regulatory Reform and judicial Review, Adm. L. Rev., vol. 48.2, Autum 1996, págs. 364 y ss.

16. ThOMAs O. SARGentich, The Critique of active Judicial Review of Administrative Agencies ; A Reevaluation «, Adm. L. Rev., vol. 49.3, Summer 1997, págs. 599-643.

17. CASS R. StINSTEIN, After the Rights Revolution. Reconceving the Regulatory State, Harvard University Press, Cambridge, Mass., 1990, pág. 67. 
nes de parte que consten en autos. En segundo lugar, en la línea de la doctrina Chevron, afirma que muchos de los principios estatutarios por los que se regulan las agencias son políticos y no jurídicos, y por tanto, en muchas ocasiones, los jueces estarían tomando ilegalmente decisiones políticas al revisar la legalidad del comportamiento de la agencia.

No debe perderse de vista que durante la Administración Bush, el President's Council on Competitiveness, dirigido por el Vicepresidente Dan Quayle, operaba como mecanismo para centralizar la revisión de la regulación de las agencias, tomando en consideración los intereses de los grandes grupos económicos cercanos al Presidente ${ }^{18}$.

El problema del control de las agencias se completa con la introducción del control de la actividad administrativa por el legislativo. El Título II del Contract with America Advancement Act, firmada por Clinton el 29 de marzo de 1996, introduce un nuevo Capítulo 8 al Título $\mathrm{V}$ del United States Code que requiere una revisión por el Congreso de las regulaciones de las agencias federales, incluidas las independientes ${ }^{19}$ : "...each final and interim final rule are required for review by Congress and to General Accounting Office (GAO) before the final or interim final rule can take effect." Las agencias, por tanto, deberán presentar a la GAO y al Congreso un report con completa documentación acerca de:

1. Análisis de los costes y beneficios de la norma.

2. Información concerniente a las acciones de la agencia bajo la Regulatory Flexibility Act (RFA). Tal ley fue sustituida en la primavera de 1996 por la Small Business Regulatory Enforcement Fairness Act, que pretende forzar el respeto y aumentar las responsabilidades de las agencias federales respecto a las pequeñas empresas, ampliando la judicial review en su subtitle $\mathrm{D}$ a un número de materias mayor que el de las contempladas por la RFA, y con un período de caducidad de la acción de un año. Parece volver así a la concepción de los años setenta del proceso administrativo como un modelo de representación de intereses, donde, de algún modo, se debía articular la participación de los grupos no representados o infrarrepresentados. Pero quizá lo único que ponga de manifiesto sea la importancia de las pequeñas y medianas empresas en el año de elecciones presidenciales (cosa que ya se advirtió en 1995 en la White House Conference on Small Business) ${ }^{20}$.

3. Información concerniente a las acciones de la agencia bajo la Unfunded Mandates Reform Act.

18 Ver Charles TIEFER, The Quayle Council : "No Fingerprints» on Regulation, en THE SEMI-SOVEREIGN PRESIDENCY: THE BUSH ADMINISTRATION'S STRATEGY FOR GOVERNING WITHOUT CONGRESS, 1994́, Págs 61-87.

19. Ver: Daniel Cohen \& Peter L. Strauss, Congressional Review of Agency Regulations, Adm. L. Rev., vol. 49.1, Winter 1997. JERry L. MAsHaw, Reinventing Govermment and Regulatory Reform : Studies in the Neglect and Abuse of Administrative Law, U. Pitt. L. Rev., vol. 57, 1996, págs 405 y ss.

20. Ver: Thomas O. SARgenTich, The Small Business Regulatory Enforcement Fairness Act, Adm. L. Rev., vol. 49.1, Winter 1997. 
4. Cualquier otra información relevante o requerida por cualquier otra ley u orden ejecutiva.

\section{LA NUEVA REGULACIÓN DE LA INSTITUCIÓN DEL HABEAS CORPUS}

La aprobación de la Antiterrorism Death Penalty Act (AEDPA) en 1996, parece reducir considerablemente las posibilidades de revisión mediante escrito de habeas corpus de las condenas de muerte dictadas por un tribunal estatal, por parte de los tribunales federales. Pretendiendo solucionar el retraso acumulado en los procesos de habeas corpus y evitar la acumulación de procesos ante instancias estatales y federales, así como crear un filtro que impidiese los escritos de habeas corpus "frívolos" o manifiestamente infundados, la AEDPA, por primera vez en la historia de los EEUU, introdujo una norma limitadora de la institución del habeas corpus.

El parágrafo 2244 AEDPA reduce la capacidad de revisión federal de las condenas capitales de los estados, a aquellos casos en que la decisión del tribunal estatal en lo referente a los méritos del reo fuera "contrary to, or involved an unreasonable application of, clearly established Federal law, as determined by the Supreme Court of the United States" o bien "resulted in a decision that was based on an unreasonable determination of the facts in light of the evidence presented in the State court proceeding".

Igualmente, la AEDPA elimina totalmente cualquier judicial review de las órdenes de expulsión dictadas por el Inmigration and Naturalization Service (INS) contra extranjeros que hayan cometido numerosos crímenes. Por último, la Illegal Inmigration Reform and Inmigrant Responsability Act de 1996, extendió la eliminación de la judicial review en su parágrafo 306 (b): “...Any final order of deportation against an alien who is deportable by reason of having committed a (covered) criminal offense... shall not be subject to review by any court, ${ }^{21}$.

En este sentido, la regulación de la AEDPA puede resultar inconstitucional por contravenir la Suspension Clause, introducida por la Enmienda catorce, ir más allá de lo permitido por la Exceptions Clause o contravenir la Supremacy Clause a que están sometidos los tribunales norteamericanos. No es este el lugar para analizar dichas posibles inconstitucionalidades, pero adelantaremos únicamente que puede interpretarse la Enmienda catorce en el sentido que sugiere el profesor Steiker ${ }^{22}$ de que al tiempo que incorpora la Suspension Clause, prohíbe la eliminación del recurso federal de habeas corpus para las sentencias capitales de los estados. Igualmente puede interpretarse como un ataque al principio de separación de poderes tal como se entiende en EEUU, dado que se produce una derogación de

21. Las citas legales han sido tomadas del articulo no firmado The Avoidance of Constitutional Questions and The Preservation of Judicial Review: Federal Court Treatment of the New Habeas Provisions, Har. L. Rev., vol. 111.6, abril 1998, págs. 1578 y ss.

2 JORDAN STEIKER, Incorporating the Suspension Clause: Is There a Constitutional Right to Federal Habeas Corpus for State Prisioners?, Mich. L. Rev., vol. 92, 1994, págs. 899-912. 
competencias del poder judicial por parte del legislativo, y es constante la jurisprudencia del Tribunal Supremo de barrera frente a la invasión por parte del Congreso de las facultades inherentes al poder judicial ${ }^{23}$.

Pero el artículo que provoca este comentario apunta un nuevo problema al analizar los casos de habeas corpus planteados ante los tribunales federales en los últimos dos años, y al poner de manifiesto el canon interpretativo de uevitación de las cuestiones constitucionales" que la Corte Suprema ha respaldado singularmente en su sentencia Felker v. Turpin de 1996, a la espera de su ratificación en el próximo caso que le será presentado: Stewart v. Martínez-Villarreal.

El autor pone de manifiesto un activismo judicial dirigido a evitar el planteamiento de las cuestiones relativas a la constitucionalidad de la AEDPA, mediante la aplicación de ciertos principios interpretativos (el llamado long-standing canon, que permite a los tribunales interpretar una ley de manera que se eviten dificultades constitucionales). Así, los jueces federales han preferido apoyar las regulaciones de la AEDPA frente a su posible inconstitucionalidad, interpretando que la ley no excluye otras fuentes de apoyo, según las cuales los tribunales federales retendrían el poder para juzgar demandas constitucionales. Dichos principios serían:

1. La cláusula general de aseguramiento de la judicial review para las regulaciones de las agencias en el ámbito administrativo, que ha sido también aplicado por los tribunales para preservar la judicial review en cuestiones de orden constitucional.

2. La presunción en contra de la revocación de competencias por órdenes implícitas en ausencia de una declaración indudable del Congreso, órdenes que pueden ser confrontadas con la aplicación de leyes paralelas.

Tales principios pueden resumirse en uno: los tribunales pueden mantener su jurisdicción y los elementos esenciales de la judicial review de cuestiones constitucionales, en ausencia de una declaración clara y terminante del Congreso en contra, principio que el autor parece valorar en beneficio de la estabilidad constitucional y la continuidad de las leyes reguladoras de la institución del habeas corpus.

\section{SELECCIÓN DE JURISPRUDENCIA ${ }^{24}$}

Se comentan, a continuación, cinco sentencias de diferentes tribunales (estatales y federales) relevantes desde el punto de vista constitucional: una relativa a separación de poderes (caso Noriega), una relativa al problema citado de la subfinanciación de las agencias (escuela pública de Ohio), una relativa a la amplitud del secreto cubierto por el executive privilege (caso del ministro de agricultura

23. Por ejemplo, últimamente: Chambers v. NASCO Inc., 501 U.S. 32, 47 (1991).

24. Se realiza a partir de la Hardvard Law Review, vol. 111. 
Mike Espy) y dos de derechos fundamentales (derechos de los convictos de delitos contra la libertad sexual y política del ejército sobre gays y lesbianas).

\section{United States v. Noriega, 117 F.3d 1206 (11th Cir. 1997)}

El General Manuel Noriega había sido acusado por el gran jurado de Florida, el 4 de febrero de 1988, de 12 cargos relativos al tráfico de drogas, su representación letrada alegó que gozaba de inmunidad diplomática y de Jefe de Estado. El Tribunal de Distrito no apreció dicha inmunidad y capturado el General Noriega el 3 de enero de 1990 por parte de una expedición militar americana, fue condenado a 40 años de prisión. El condenado recurrió en apelación ante el Eleventh Circuit. No existiendo norma legal sobre quién es el llamado a determinar la condición de jefe de estado extranjero y su consiguiente inmunidad, los tribunales suelen remitir su decisión al ejecutivo, en la medida en que las relaciones exteriores, conforme al sistema de división de poderes norteamericano, corresponden a dicho poder. Dicha capacidad no ha sido nunca contestada por ningún tribunal, existe sin embargo, una jurisprudencia más confusa cuando falta indicación concreta por parte del ejecutivo. En In re Doe (860 F.2d 40 (2d Cir. 1988)), el Second Circuit resolvió que en ausencia de dicha indicación procedía denegar la inmunidad, el Fifth Circuit, sin embargo, en Spacil v. Crowe (489 F.2d 614 (5th Cir. 1974) sostuvo que, en tal caso, los tribunales "debían realizar una determinación independiente de la inmunidad"25. En la sentencia objeto de reseña, el Eleventh Circuit sostuvo una posición en cierto modo intermedia al resolver que también en ausencia de indicación explícita del Ejecutivo, debía buscarse una indicación implícita del mismo por parte del Tribunal, indicación que, en el caso concreto, provendría de su secuestro por parte de las tropas norteamericanas. Según los comentaristas de la Hardvard Law Review ${ }^{\mathfrak{y}_{1}}$ esta actitud del Eleventh Circuit resulta contraria a la división de poderes establecida en la Constitución en la medida en que abre una posibilidad de interpretación de las actividades del ejecutivo en política exterior (las indicaciones implícitas o no verbales), que, además, puede conducir a resultados contrarios a los deseados por el Ejecutivo. Igualmente, consideran esta solución contraria a la división de poderes en la medida que implica que el Tribunal debe decidir, primero, quién es el Jefe de Estado extranjero, reconocimiento que, a su juicio, corresponde al Ejecutivo. Es de destacar que no se haya suscitado comentario alguno sobre la constitucionalidad del secuestro por el Ejército como mecanismo procesal.

\section{DeRolph v. State, 677 N.E. 2d 733 (Ohio 1997)}

En 1991, diferentes sectores de la educación (padres, escuelas, etc.) trataron de obtener la declaración de inconstitucionalidad del sistema de financiación de

25. Otros tribunales han sostenido la regla contraria, en ausencia de indicación, se concede la inmunidad, por ejemplo, Lafontant $v$. Aristide, 844 F. Supp. 128, 134 (E.D.N.Y. 1994).

za Vol. 111 , pägs. 855 y ss. 
la escuela en Ohio, el Tribunal del Condado apreció su demanda. El Tribunal de Apelación del Quinto Distrito sostuvo la opinión contraria y, finalmente, el Tribunal Supremo de Ohio declaró la inconstitucionaldiad de dicho sistema.

El Tribunal Supremo de los Estados Unidos en San Antonio Independent School District v. Rodríguez 411 U.S. 1 (1973) sostuvo que la educación no es un derecho constitucional federal fundamental, por lo que no era posible buscar ante dicho Tribunal amparo frente a sistemas de financiación escolar no equitativos. Algunos estados, sin embargo, sí han reconocido la existencia de un derecho constitucional estatal a la educación. La Constitución de Ohio garantiza un sistema «tbrough and efficient, de escuelas en todo el Estado. El Tribunal Supremo de Ohio mantuvo en la sentencia citada que, dadas las deficiencias apreciadas en las escuelas, dicho sistema no satisfacía tales exigencias y que los encargados de dar cumplimiento al mandato constitucional eran tanto el legislativo como el judicial. El Tribunal ordenaba, por tanto, a la General Assembly que estableciera un nuevo sistema de financiación estatal de las escuelas en el plazo de un año. Esta sentencia tuvo dos votos particulares en los que, básicamente, se sostuvo el carácter político de la cuestión y, por tanto, su condición de no justiciable. La decisión del Tribunal aunque generalmente bien recibida, ha sido criticada por no haber definido el parámetro de tbrough and efficient, lo que podría hacer inútil todo el proceso, si el nuevo procedimiento aprobado se muestra tan incapaz como el anteriormente existente.

\section{In re Sealed Case, 116 F.3rd 550 (D.C. Cir. 1997)}

Este caso surge como consecuencia de una investigación de la Office of the Independent Counsel (OIC) en relación con las presuntas gratificaciones ilícitamente recibidas por el Ministro de Agricultura Mike Espy, como consecuencia de las actuaciones de su Ministerio ante terceros. Emitido un mandamiento de aportación de documentos (subpoena duces tecum) al Counsel to the President por parte del Gran Jurado, la Casa Blanca aportó parte de la documentación de su investigación interna y retuvo varios documentos alegando "executive/deliberative privilege". Revisados finalmente in camera por el tribunal del distrito, se apreció el privilegio alegado por la Casa Blanca. El D.C. Circuit resolvió la apelación de la OIC según la cual el privilegio sólo cubriría los documentos enviados o recibidos por el Presidente, lo que no era el caso. El Tribunal apreció la existencia del privilegio pero ordenó que se diera a la OIC la oportunidad de demostrar que dichos documentos eran relevantes penalmente y no podían ser obtenidos con la debida diligencia de otra fuente, de manera individual. Es decir, que el Tribunal ha extendido el Privilegio de las Comunicaciones Presidenciales reconocido por primera vez en United States v. Nixon 418 U.S. 683 (1974) no sólo a aquellas en que participa el Presidente, sino también a documentos que el Presidente nunca había visto, pero que se referían a los Consejeros del Presidente. Esta decisión, por tanto, iguala las comunicaciones del Presidente (elegido democráticamente y, por ello, sometido a responsabilidad política directa) con las de sus consejeros. Esta exten- 
sión se ha considerado injustificada en la medida en que los altos funcionarios de la Casa Blanca ya vienen cubiertos por el deliberative process privilege y por las excepciones a la revelación de la Freedom of Information Act [5 U.S.C. $₫ 552$ (1994)].

\section{E.B. v. Verniero, 119 F.3d 1077 (3d Cir. 1997)}

El 31 de octubre de 1994, New Jersey aprobó la Registration and Notification of Release of Certain Offenders Act ${ }^{27}$ (Ley de Registro y Notificación de la Puesta en Libertad de Determinados Delicuentes), comúnmente denominada Ley Megan. En ella se exige que los convictos de delitos contra la libertad sexual se inscriban, a su excarcelación, en un registro y que se comunique su presencia a aquellos miembros de la comunidad que puedan entrar en contacto con los mismos, con el fin, se alega, de prevenir posibles nuevos delitos. El Tercer Circuito en Artway $v$. Attorney General 81 F.3d 1235 (3d Cir. 1996) sostuvo la constitucionalidad de la obligatoriedad del registro. En enero de 1996, diferentes afectados plantearon la inconstitucionalidad de la norma (en lo relativo a la notificación) alegando que infringia el principio de non bis in idem punitivo y suponía una reformatio in peius (double jeopardy and ex post facto clauses) (principio de legalidad penal). El Tribunal, no apreció estas circunstancias y decretó la constitucionalidad de la norma. Su razonamiento seguía el establecido en la sentencia citada y, en esencia, reducía -correctamente- el problema a la determinación de si la notificación a la comunidad de su condición de convicto excarcelado por cumplimiento de su condena supone o no una "pena" en sentido legal. A tal efecto, consideraba el propósito del legislador, la finalidad objetiva de la norma y el efecto de la misma. De manera bastante sorprendente, el Tribunal alcanza la conclusión de que, en ninguno de los tres casos, cabía concluir que se tratara de una pena. Pese a que la aprobación de la norma se produjo como consecuencia de un caso concreto de reincidencia y contra dicho tipo de delincuentes, el Tribunal consideró que la notificación se realizaba con fines de prevención y rehabilitación social (mejora del control del condenado). Igualmente, aunque existen notables semejanzas entre este procedimiento y muchos otros de "vergüenza pública", "picota", etc., el Tribunal no apreció que se tratara de un castigo e insistió en la finalidad objetiva de prevención. El Tribunal tampoco apreció que la "exclusión social" (que se deriva de la notificación) es la base misma de cualquier forma de castigo organizado. Por último, aunque en todos los casos en que se había producido el conocimiento por otros medios de la condición de ex convicto en la comunidad, se habían producido consecuencias dañosas (pérdida de empleo, expulsión de asociaciones, etc.), el Tribunal no consideró que el efecto de la norma fuera una "pena". Al no considerar que se trataba de una pena, no procedian -a juicio del Tribunal- las cuestiones relativas al non bis in idem punitivo, ni a la reformatio in peius. Cuestiones

27. N.J. Stat. Ann. $\ 2$ C:7-1 a 7-11 (West 1997). 
éstas absolutamente relevantes puesto que, no hay que olvidarlo, se trata de delincuentes que ya ban satisfecho la pena que se les ba impuesto y que, por tanto, ven reforzado su castigo, después del juicio.

\section{California Army National Guard, 124 F.3d 1126 (9th Cir. 1997)}

El Congreso aprobó en 1993 la política denominada "no preguntes, no lo cuentes" (don't ask, don't tell) en sustitución de la anterior política del Departamento de Defensa de expulsión de todos los homosexuales del ejército. Esta nueva política dio lugar a la consiguiente directiva del Departamento de Defensa (1332.30 (1994)), según la cual "La orientación sexual de cada miembro se considera un asunto privado y personal, y no constituye un impedimento para continuar en el servicio según el presente artículo, salvo que se manifieste en forma de conducta homosexual. Los actos homosexuales, por tanto, siguen prohibidos (10 U.S.C. $\$$ 654(b)(1) (1994)) y, aun más, quienes manifiesten su condición homosexual serán expulsados del ejército salvo que puedan demostrar la ausencia de tales conductas ("Cualquier miembro de las fuerzas armadas será separado del servicio ... si ... dicho miembro manifiesta ser un/una homosexual o bisexual, o palabras que lo impliquen, salvo que pueda averiguarse ... que dicho miembro ha demostrado que no se trata de una persona que participe, trate de participar, tenga propensión a participar, o se proponga participar en actos de homosexualidad").

En esta sentencia, el Noveno Circuito se suma a la opinión unánime del resto de tribunales federales (Tercero, Cuarto y Octavo) en su consideración de que dicha regulación no infringe la cláusula de igualdad, ni la Primera Enmienda («El Congreso no dictará ley alguna ... que coarte la libertad de palabra o de imprenta"). El Primer Teniente Charles Holmes, miembro de la Guardia Nacional del Ejército de California envió un escrito al oficial al mando del CANG comunicándole que upor razones de conciencia, honestidad y orgullo, me veo obligado a comunicarle que soy homosexual. En la audiencia subsiguiente el oficial se negó a destruir la presunción de que podría participar en actos homosexuales, por lo que fue separado del servicio. Aunque el Tribunal del Distrito apreció la violación de la cláusula de igualdad y de la Primera Enmienda, el Noveno Circuito casó su sentencia. El Tribunal afirmó que existía un interés legítimo en excluir del servicio a quienes realizan actos de homosexualidad para mantener la disciplina y la disposición al combate, y que la afirmación de ser homosexual constituye una indicación suficientemente racional de la disposición a realizar tales actos. Igualmente, el Tribunal argumentó que la separación del servicio no se producía exclusivamente por su afirmación sino por su negativa a destruir la presunción creada por su afirmación. Es decir, que la separación del servicio no se produce por su condición de homosexual (permitida), sino por la uinferencia de una conducta homosexual" (prohibida). La ley no violaría la Primera Enmienda porque no castigaba la expresión, sino la conducta.

En voto particular el Juez Reinhardt consideró que la norma violaba la Primera Enmienda ya que, admitiendo que la conducta homosexual pudiera ser 
constitucionalmente castigada, no se deduce que pueda castigarse la afirmación de la condición de homosexual. El argumento de que la afirmación se utiliza sólo como prueba de la conducta prohibida es incorrecto, toda vez que la afirmación de la homosexualidad no es causa de expulsión según esta misma política. El Juez Reinhardt afirma, igualmente, la falacia que encierra la distinción entre condición y conducta homosexual, y que, en todo caso, la política supone una restricción ilegítima a la libertad de expresión ("comunicar lo más íntimo de sus emociones y de su identidad a los demás").

En este mismo sentido, la diferenciación entre expresión y conducta resulta falaz (incluso admitiendo que sea posible) en la medida en que para establecerla se obliga a destruir una presunción que es, de hecho, indestructible, puesto que implica demostrar que no existe una predisposición a realizar actos en el futuro, demostración que es, por su propia naturaleza, imposible.

Esta política, por último, es contraria a la libertad de expresión en, al menos, dos sentidos. En primer lugar, impide que los valores homosexuales se incorporen a los valores culturales genéricos del ejército, restringiendo ilícitamente los componentes que integran la identidad cultural del ejército y sus miembros. En segundo lugar, estimula la ocultación y el engaño como preferibles a la libre expresión de la identidad personal, desvalorizando, de este modo, la libertad de expresión que debiera ser protegida tanto en su faceta permisiva como axiológica. 